\title{
Cross-sectional Capacitance Measurement of Particle Concentration in a Microchannel with Multi-layered Electrodes
}

\author{
Nur Tantiyani Ali Othman* ${ }^{\mathrm{a} 1}$, Hiromichi Obara ${ }^{\mathrm{b} 2}$, and Masahiro Takei ${ }^{\mathrm{a} 3}$ \\ ${ }^{a}$ Graduate School of Mechanical Engineering, Division of Artificial System Science, Chiba \\ University, 1-33 Yayoi, Inage, Chiba 263-8522, Japan \\ Tel \& Fax: +81-(0)43-290-3212 \\ Email: ${ }^{1}$ tantiyani@yahoo.com, ${ }^{3}$ masa@ chiba-u.jp \\ ${ }^{b}$ Department of Mechanical Engineering, Graduate School of Science and Engineering, Tokyo \\ Metropolitan University, 1-1 Minamioosawa-Osawa, Hachiouji 192-0397, Japan \\ Tel \& Fax: +81-(0)42-677-2943 \\ Email: ${ }^{2}$ obara@tmu.ac.jp
}

\begin{abstract}
:
In the present study, cross-sectional capacitance is measured in a microchannel with 12 multi-layered electrodes. The cross-sectional capacitances of microparticles of three different diameters are measured upstream and downstream. Based on these measurements, the particle concentration in term of particle volume fraction is calculated. The particle volume fraction at three configuration areas of electrode pairs, namely, at ones containing adjacent, horizontal, and vertical pairs, are considered for ease of understanding of the particle concentration for different electrode pairs. The particle volume fraction shows an increment of 1.0 to $9.3 \%$ for electrode pairs near the channel walls, but decreased by 0.4 to $0.96 \%$ for center electrode pairs from the upstream cross-section to the downstream cross-section. Moving from the inlets to the outlet, the particles drift away from the center of the microchannel and approach the channel walls and the number of particles that migrate toward the channel walls increases clearly in the outlet area.
\end{abstract}

Keywords: Cross-sectional capacitance; microchannel; multi-layer electrodes; two-phase flow; particle volume fraction

\section{Introduction}

Microfluidic miniature devices have received significant attention over the past few decades because of their wide applicability to scientific and engineering applications in industries such as life sciences and combinatorial synthesis [1]. Microchannel devices have been shown to be very effective for handling particles with increased control and sensitivity due to the possibility of shortening the diffusion distance of molecules in a narrow space, which can reduce the reaction time, and to a large specific interfacial area and low heat capacity, which enables rapid heating and cooling. These advantages make the microchannel an efficient device for the measurement of the distribution of microparticle concentration in the vertical cross-sectional microchannel.

Microparticle concentration distribution measurement has become a fundamental research topic because the particle concentration in the microchannel is nonlinear and heterogeneous, and unsteady with respect to time. The determination of the particle concentration distribution is very important in order to enhance the efficiency of the process, which relies on the effective performance and rapid separation of particles. The particle concentration distribution is determined in terms of the particle volume fraction as the ratio of the volume occupied by particles to the total volume of the fluid carrier in the microchannel. The particle concentration distribution reveals the affinity of particles to transport and move transversely 
in the microchannel is referred to as particle migration. Normally, the ratio of the microchannel size to the particle size is relatively large and the Reynolds number $(\mathrm{Re})$ is small. Under such flow conditions, particles do not follow the fluid streamlines. Therefore, particle migration is frequently observed [2], and the particle migration in the fluid carrier is often subjected to an inertial lift force. As a result, the particles acquire a component of velocity that is transverse to the flow streamlines. The transverse motion, although weaker than the streamwise particle motion, plays an important role in microfluidic processes, such as deposition and suspension of particle [3].

Phenomena related to particle migration have gained a great deal of attention since early 1962, with numerous studies on large-scale particle migration. The first experiment on particle migration in cylindrical Poiseuille flow was a study by Segre and Silberberg [4]. They discovered that neutrally buoyant spherical particles in a pipe flow of a Newtonian fluid migrate to a radial position of about 0.6 times the tube of channel, in other words they found the existence of an equilibrium height in the channel where the particles tend to migrate. Particle migration in dilute suspensions has been extensively and systematically investigated since Segre and Silberberg observed that particles accumulate at certain equilibrium positions. Their study triggered a series of experimental and theoretical studies on particle migration in tube flow [5], channel flow [6], and Couette flow [7] involving neutrally-buoyant particles $[8,9]$ and non-neutrally-buoyant particles [10]. Furthermore, a study on particle migration at equilibrium positions in circular pipes or planar channels with different Reynolds numbers (Re) [11] using a regular perturbation method at low $\operatorname{Re}[12,13]$, in a straight microchannel with four electrode pairs [14], through a square microchannel [3], and using a dynamic self-assembly process [15].

The aforementioned studies used a micro-PIV laser technique to measure the behavior of particle migration in two-phase flows. However, the micro-PIV laser technique is expensive and limits the particle concentration to dilute particle concentrations. Capacitance measurement may overcome this limitation on particle concentration [16]. Few studies on particle migration based on capacitance measurement have been conducted. Griffiths et al. [17] developed a circular array with microelectrodes for particle migration measurement in a miniature tomography application. York et al. [18] used microelectrodes in a miniature sensor to visualize the particle distribution in a single cross-section. Choi et al. [19] estimated the sub-micro-particle movement and the spatial transition of the particle concentration distribution for different cross-sections. Jaworek et al. [20] investigated the phase shift using a capacitance sensor and found the phase shift to be directly proportional to the particle volume fraction.

However, the characteristics of particle migration in a microchannel with 12 multi-layered electrodes using a capacitance measurement system remain unclear. Moreover, there have been few studies on the particle concentration distribution at different vertical cross-sections for electrode pair configurations. Therefore, the objective of the present study is to determine the particle concentration distribution for different electrode pairs and cross-sections, for adjacent electrode pairs, at the right and left sides of a vertical electrode pair, and at the upper and lower sides of a horizontal electrode pair based on the cross-sectional capacitance measurement. In addition, the particle migration behavior from the upstream cross-section to the downstream cross-section is discussed in detail.

\section{Experiments}

\subsection{Experimental set- up}

The experimental equipment is set up consists of two micro-pumps, a microchannel, a multiplexer, a capacitance measurement system, and a computer, as shown in Fig. 1. Two micro-pumps (IC3100 [KDS100], KD Scientific, USA) are used as an automatic dispenser and control the flow rate to the microchannel inlets. The microchannel consists of five vertical cross-sections, a sensor electrode, a silica substrate, and a glove. Choi fabricated a microchannel system using micro-electro-mechanical-systems 
(MEMS) technology. In-depth information on the microchannel fabrication procedure can be obtained from Choi et al. [21].

The cross-sectional capacitance is measured at upstream and downstream cross-sections. The length of the microchannel from the origin to the outlet is $20 \mathrm{~mm}$. The upstream cross-section is located in the center of the microchannel at $z=10 \mathrm{~mm}$, and the downstream cross-section is located near the outlet at $z$ $=20 \mathrm{~mm}$, as shown in Fig. 1. The multiplexer charges a frequency and voltage; acts as a switch. The capacitance measurement system (PTL 300E, Agilent Technologies, Inc., USA) was used to measure the cross-sectional capacitance between connected electrode pairs and send the cross-sectional capacitance measurement to the computer.

In the present study, the cross-sectional microchannel is diamond shaped. Fig. 2(a) shows a micro-image of the cross-sectional microchannel captured by high-speed micro-computed tomography (XT H 225, Nikon Technologies, Inc. Japan), and a close up of the microchannel is shown in Fig. 2(b). Fig. 2(c) shows a schematic diagram of the cross-sectional microchannel. The height and width of the cross-sectional microchannel are $800 \mu \mathrm{m}$ and $700 \mu \mathrm{m}$, respectively. Each cross-section is embedded with 12 multi-layered sensor electrodes and is connected to the capacitance measurement system. The length and height of the sensor electrode are $200 \mu \mathrm{m}$ and $0.25 \mu \mathrm{m}$, respectively. The sensor electrode material is platinum, and the microchannel substrate is quartz glass.

\subsection{Experimental conditions}

Polystyrene particles (Duke Scientific Co., USA) are used as dielectric particles, and deionized water $(2 \mathrm{M} \Omega)$ is used as a non-conductive liquid. The resistivity of deionized water is $1.8 \times 10^{5} \Omega \mathrm{m}$ and that of the polystyrene particles is $2.8 \times 10^{5} \Omega \mathrm{m}$. Particles of $1.3 \mu \mathrm{m}, 1.5 \mu \mathrm{m}$, and $2.1 \mu \mathrm{m}$ in diameter with a coefficient of variance (maximum $\mathrm{CV}$ ) of $\leq 10 \%$. The particle density, $\rho_{p}$, is $1.05 \mathrm{gcm}^{-3}$, which is close to the density of deionized water, in order to avoid unwanted settling of particles. The initial concentration of a $3 \%$ particle solution (particle: $3 \mathrm{ml}+$ deionized water: $97 \mathrm{ml}$ ) is injected through the inlets with a total inlet volume flow rate, $Q$, of $0.001 \mathrm{mLs}^{-1}$ and a spatial averaged particle velocity flow, $v$, of $0.25 \times 10^{-3} \mathrm{~ms}^{-1}$.

The capacitance measurement system operates at a switching frequency of $12.5 \mathrm{MHz}$ at 4.5 volts, which allows capacitance measurement values of down to $0.1 \mathrm{fF}$ to be resolved. The time measurement of the cross-sectional capacitance, $T$, is the number of sampling data, $N$, times the time measurement for one full frame of 66 electrode pairs, $\Delta t$. Here, $N$ is 50,000 and $\Delta t$ is $20 \mathrm{~ms}$. To get the accurate data of the capacitance measurement, the same experiment condition and method of the cross-sectional capacitance measurement is repeated in 10 deviation times, and from that 10 deviation times, the time-averaged cross-sectional capacitance is calculated.

\subsection{Experimental methods}

The experiment is carried out by separately injecting deionized water and particles through inlets A and B using micro-pumps, and the cross-sectional capacitance is measured at different cross-sectional positions, as shown in Table 1. In the first experiment, in case 1, flow deionized water is injected, and the cross-sectional capacitance at 66 electrode pairs is measured at the upstream and downstream cross-sections. The second experiment for case 2 is injected with a $3.0 \%$ particle solution in deionized water, and the cross-sectional capacitance is measured at the upstream and downstream cross-sections. The experiment is repeated for three particle sizes $(1.3 \mu \mathrm{m}, 1.5 \mu \mathrm{m}$, and $2.1 \mu \mathrm{m})$ and the particle volume fraction is calculated based on the cross-sectional capacitance measurement.

In the capacitance measurement of 12 connected electrodes, the first electrode, electrode 1, is selected as a source electrode, $i$, and electrodes 2 through 12 acted as the detecting electrode, $j$. Electrode 1 is energized by applying a voltage and the induced charge is measured from electrodes 2 through 12. Then, electrode 2 is energized, and capacitance measurements from electrodes 3 through 12 are 
performed. This process is continued until electrode 11 is energized, and the measurement is taken from electrode 12. The parallel measurements are independent because the capacitances are determined from the charge/discharge circuit through the detecting electrodes, which are all held at virtual earth potential. The 11 parallel channel outputs are selected one by one for further amplification and conversion [22]. In total, there are $N(N-1) / 2$ independent measurements, where $N$ is the number of electrodes. For the 12 electrodes, there are 66 single electrode combinations. Therefore, there are 66 independent cross-sectional capacitance measurements, as shown in Table 2. Note, the electrode pairs shown in italics in Table 2 are adjacent electrode pairs, and the electrodes pairs shown in bold are vertical electrode pairs. The electrodes pairs shown in underlined text are horizontal electrode pairs. These electrode pairs are used in further calculation of the particle volume fraction.

The 12 multi-layered capacitance electrodes are connected to the capacitance measurement system using electronic switches and wires, such as coaxial cables. The sensor electrodes may have signal capacitances in the fractional picofarad $(\mathrm{pF})$ range, and connecting to these electrodes with a $60 \mathrm{pF}$ per meter coaxial cable could totally obscure the signal. However, with correct shielding of the coaxial cable as well as any other stray capacitance one can almost completely eliminate the effects of noise. In addition, when the capacitance measurement system is operated at high frequency, the stray capacitance and residual inductances will inherent to the cables, electrical leads, and other portions of the measurement circuit that can reduce the sensitivity of stray capacitance. This time variation of sensitivity leads to beats and aliasing where noise at frequencies which are integral multiples of the oscillator frequency is aliased down to a low frequency. All of these components and connections affect the cross-sectional capacitance measurement between the electrode pairs because they generate the stray capacitance, which can degrade the measurement accuracy. It is because the cross-sectional capacitance measurement at each electrode pair will become larger than the actual cross-sectional capacitance value because an additional measurement is included as the stray capacitance effect between the measurements of the electrode pairs as follows:

$$
\begin{aligned}
& C^{L 0}{ }_{i-j}=C^{L 0^{*}}{ }_{i-j}+C^{\prime}{ }_{i-j} \\
& C^{P 0}{ }_{i-j}=C^{P 0^{*}}{ }_{i-j}+C^{\prime}{ }_{i-j} \\
& C^{P}{ }_{i-j}=C^{P^{*}{ }_{i-j}+C^{\prime}{ }_{i-j}}
\end{aligned}
$$

where $C$ is the capacitance, the superscript $L O$ indicates flow deionized water, the superscript $P O$ indicates static particles, the superscript $P$ indicates flow particles, the superscript $*$ indicates an actual value, and the prime (') indicates the stray effect. The order to the cross-sectional capacitance between the pair of electrodes varies from less than $1 \mathrm{fF}, 10^{-15}$, to a few picofarads, $10^{-12}$, whereas the stray capacitances are in the range of hundreds of picofarads. Therefore, in calculating the particle volume fraction, we consider the stray capacitance effect in order to avoid signal loss and measurement errors. This problem can usually be handled with shields and careful power supply decoupling.

\section{Experimental results}

The time-averaged cross-sectional capacitances of 66 electrode pairs are measured for different particle diameters at the upstream and downstream cross-sections. Fig. 3 shows a comparison of the calibration cross-sectional capacitance of flow deionized water, $C^{L O}{ }_{i-j}$, and $1.3 \mu \mathrm{m}$ static particles, $C^{P 0}{ }_{i-j}$, for 66 electrode pairs at the upstream and downstream cross-sections. The experiment condition is such that the inlets are injected with a low-dielectric material (particles) under a static condition and are then injected with a high-dielectric material (deionized water) in a flow condition.

The experimental results for each electrode pair show different cross-sectional capacitances because cross-sectional capacitance depends on the distance between the electrode pair. The distance between the 
pair of electrodes is inversely proportional to the cross-sectional capacitance. Adjacent electrode pairs, such as electrode pairs 1 (1-2) and 12 (2-3), have higher cross-sectional capacitance, approximately 1,100 $\mathrm{fF}$, as compared to the other electrode pairs, whereas oppositely arranged electrode pairs, such as electrode pairs 20 (2-11) and 28 (3-10), have the lowest cross-sectional capacitance. From Fig. 3, the calibration of the cross-sectional capacitance measurement for deionized water is almost the same and does not differ greatly at the upstream and downstream cross-sections. This figure shows that the cross-sectional capacitance for flow deionized water is constant and independent of the time, even for different cross-sections. The calibration of the cross-sectional capacitance measurement for static particles differs at the upstream and downstream cross-sections and is much lower than that of deionized water due to the low dielectric permittivity of polystyrene particles, as compared to deionized water, and due to the surface interaction between particles. The discrepancy of the calibration cross-sectional capacitance for deionized water and static particles does not vary greatly, because of the low particle concentration solution.

Fig. 4 shows the time-averaged cross-sectional capacitance for flow particles at three particle sizes at the upstream and downstream cross-sections. The experimental results show that the time-averaged cross-sectional capacitance for $2.1 \mu \mathrm{m}$ is up to $4.0 \%$ higher than for the other particle sizes at the upstream and downstream cross-sections, which indicates that the cross-sectional capacitance is proportional to the particle size. The larger particle sizes have higher cross-sectional capacitances. In addition, the time-averaged cross-sectional capacitance at the downstream cross-section is $1.6 \%$ greater than that at the upstream cross-section for all particle sizes because the particles have sufficient time to distribute in the fluid flowing from the upstream cross-section to the downstream cross-section.

\section{Discussions}

By assuming that a virtual capacitance of the electrical circuit is connected in parallel, the permittivity distribution of a two-phase dielectric material medium can be measured as follows:

$$
\varepsilon_{i-j}=\frac{C_{i-j} d_{i-j}}{A}
$$

where $\varepsilon$ is the permittivity distribution, $d$ is the distance between the pair of electrodes, and $A$ is the area of the sensor electrode. The permittivity distribution is a measurement of the ability of particles to respond and to be transmitted to an electric field, which is generated by the sensor electrodes. Table 3 summarizes the distance between the pair of electrodes. The distance between a pair of adjacent electrodes, such as electrode pair 1-2, is $141.4 \mu \mathrm{m}$, whereas the distance between a pair of oppositely arranged electrodes is $687.5 \mu \mathrm{m}$ for electrode pair 2-8 and $725.0 \mu \mathrm{m}$ for electrode pair 1-9. The area of the sensor electrode, $A$, is $5.0 \times 10^{-11} \mathrm{~m}^{2}$.

Based on the cross-sectional capacitance measurement and the permittivity distribution, the particle volume fraction is determined for different electrode pair areas. There are many methods for determining the particle volume fraction based on the permittivity distribution. One such method involves the use of the Maxwell equation [24], which is widely used and derived from a self-consistent model by solving the Laplace equation. Therefore, in order to measure the particle volume fraction based on the cross-sectional capacitance measurement, we modified the Maxwell equation as described by Louge et al. [24] for simple measurement of the particle volume faction, including the effect of curvature in the velocity distribution and assuming immunity to the stray capacitance. The particle volume fraction, $(1-\phi)_{i-j}$, for different electrode pairs is calculated by rearranged the Maxwell equation as follows: 


$$
\left.(1-\phi)_{i-j}=\xi \frac{\left(\varepsilon^{P}{ }_{i-j}-\varepsilon^{P 0}{ }_{i-j}\right.}{\left(\varepsilon^{L 0}{ }_{i-j}-\varepsilon^{P 0}{ }_{i-j}\right.}\right)
$$

where $\xi$ is the maximum particle volume fraction equivalent to the initial particles concentration. To verify that the position of the vertical cross-section and electrode pair area bears little effect on the particle volume fraction distribution, we prepared a simple electrode pair configuration, focusing on three electrode pair areas, is arranged. Fig. 5 shows the configuration of the electrode pair with the origin at center the $x$ - and $y$-axes of the diamond-shaped cross-sectional microchannel.

Measurement is performed for eight adjacent electrode pairs 1-2, 2-3, 4-5, 5-6, 7-8, 8-9, 10-11, and 11-12. Vertical electrode pairs are measured for oppositely arranged electrode pairs at left-hand and right-hand sides of the cross-sectional microchannel. Six vertical electrode pairs are measured in the vertical pair area, where electrode pairs 3-4, 2-5, and 1-6 are on the left-hand side and electrode pairs 7-12, 8-11, and 9-10 are on the right-hand side. The horizontal electrode pair is measured for oppositely arranged electrode pairs on the upper and lower sides of the cross-sectional microchannel. Six electrode pairs are measured in the horizontal pair area, namely, electrode pairs 1-12, 2-11, and 3-10 on the upper side and electrode pairs 4-9, 5-8, and 6-7 on the lower side.

The particle volume fraction can be expressed as the tendency of particles to migrate and transport to different area. Figs. 6 through 8 shows the particle volume fraction for three particle size at the upstream and downstream cross-sections for adjacent, horizontal, and vertical electrode pairs, respectively. The horizontal axis is the distance between the vertical and horizontal electrode pairs from the center of the microchannel. The value of the particle volume fraction ranges between 0 and 0.03 , where 0 indicates only a single phase of the flow deionized water is present and 0.03 indicates that all particles are dominant in the area of the electrode pair measurement.

The particle volume fraction for adjacent pairs is relatively high, approximately $0.03 \%$, at both the upstream and downstream cross-sections. In addition, the same pattern appears at the upstream and downstream cross-sections. This pattern shows that the particles tend to migrate and concentrate near the channel walls. In addition, the particle volume fraction is proportional to the particle size, where the particle volume fraction for $2.1 \mu \mathrm{m}$ is up to $0.31 \%$ greater than that for the other smaller particles sizes. This is because the larger particles induce a sufficient inertial lift force due to the high inter-surface interaction area with a high velocity distribution near the walls, which pushes and drives particles, allowing easier movement and transport in the flowing fluid.

The particle volume fraction for the vertical pair at the upstream and downstream cross-sections is shown in Fig. 7. The particle volume fraction at the upstream cross-section increases gradually from the vertical electrode pair near the walls (electrode pairs 3-4 and 9-10) to the vertical electrode pair near the center of the microchannel (electrode pairs 2-5, 8-11, 1-6, and 7-12) at both the left-hand and right-hand sides of the microchannel for all particle sizes. As shown in the figure, for the upstream cross-section, higher particle volume fractions occur at the electrode pairs near the center of the microchannel._In contrast, the particle volume fraction at the downstream cross-section decreases gradually from the vertical electrode pair near the channel walls to the vertical electrode pair at the center of the microchannel at both the left-hand and right-hand sides of the microchannel for all particle sizes. This indicates that, initially, particles are much more highly concentrated at the center of the microchannel, and that the particles moved slowly from the center of the microchannel to an equilibrium position near the walls as the particles moved from the upstream cross-section to the downstream cross-section. The particle volume fraction for electrode pairs near the walls increased by 7\% for electrode pair 3-4 and by $4 \%$ for electrode pair 9-10. In contrast, the particle volume fraction for electrode pairs near the center of the microchannel decreased up to $0.5 \%$ from the upstream cross-section to the downstream cross-section for electrode pairs 1-6 and 7-12. Its indicates that more particle migration is observed at electrode pairs at 
the walls vicinity area than at those near the center as particles moved from upstream to downstream cross-section.

The same particle volume fraction pattern as at the vertical pair appears, where on the upper side, the particle volume fraction is higher for electrode pair 1-12 and decreases gradually for electrode pairs 2-11 and 3-10. Similarly, the particle volume fraction increased gradually from electrode pair 4-9 to electrode pairs 5-8 and 6-7 near the walls. The particle volume fraction near the channel walls increased up to $9 \%$ for electrode pairs 1-12 and 6-7 as the particles move from the upstream cross-section to the downstream cross-section. However, the particle volume fraction at electrode pairs near the center of the microchannel decreased less than $1 \%$ for electrode pairs 3-10 and 4-9. Its indicates that more particle migration is observed at electrode pairs at the walls vicinity area than at those near the center as particles moved from upstream to downstream cross-section. This is because the inertial lift forces dominate the particle migration when $\mathrm{Re}$ is small. The presence of the inertial lift forces keep the particles from remaining in a stationary position and cause the particles to migrate away from their original position. The inertial lift forces push the particles away from center of the microchannel to an equilibrium position. The particle equilibrium position is obtained as a function of $\mathrm{Re}$, and the critical $\mathrm{Re}$ at the particle equilibrium position starts to increase. The spatial distribution of the particle volume fraction reveals that marked particle migration occurs even at low Re in the microchannel, which is induced by the high shear inertial lift force rate due to small-scale effects.

In addition, the particle migration mechanism that causes the particles to migrate toward the channel walls is based on the curvature of the velocity profile, and the particles are assumed to move with an angular velocity. The neutral buoyancy of the particles that move parallel to the streamlines when the fluid velocity relative to the particles is larger at the channel walls compared to the center of the microchannel. The parabolic nature of the laminar velocity profile also contributes to the shear-induced inertial lift force, which drives particles away from center of the microchannel toward the walls. As the particles migrated closer to the walls, an asymmetric wake induced around the particle generates a wall-induced inertial lift force and drives the particles away from the walls [14]. From the parabolic velocity profile, a low gradient of the particle velocity at the center of the microchannel pushes the particles toward the walls, while a high gradient of the particle velocity near the walls drives the particles back to the center of the microchannel.

The calculation of the particle volume fraction for all electrode pairs shows that at the upstream cross-section, particles are much more highly concentrated than at the electrode pairs near the center of the microchannel and have the lowest concentration near the walls. In contrast, the particle volume fraction at the downstream cross-section is more highly concentrated near the channel walls. This is because, at the upstream cross-section, the wall-induced inertial lift force is dominant and pushes the particles toward the center of the microchannel. Moreover, due to the wall-induced inertial lift forces and the gradient of the velocity distribution, the particles moved and migrated to an equilibrium position. The balance between these forces finally results in the equilibrium of the particle migration in the microchannel.

The particle volume fraction for three area measurements in the cross-sectional microchannel can be summarized as shown in Fig. 9, which shows the tendency for particles to migrate due to the inertial lift forces at the upstream and downstream cross-sections. These inertial lift forces are a combination of the shear-induced inertial lift force $\left(F_{S L}\right)$, which push particles toward the walls, and the wall-induced inertial lift force $\left(F_{W L}\right)$, which drive particles towards the center of the microchannel [15]. It can be seen that the parabolic flow distribution across different $z$-axis positions next magnifies the interparticle spacing. That is, particles are pushed apart due to the parabolic velocity distribution. The particle moves closer to center streamlines when it interacts in this repulsive phase. The particles however, do not move off to infinity but are pushed back toward their focusing positions and trajectories are stabilized by the lift forces. The multiple oscillation cycles to reach the equilibrium focusing positions can be explained by overshooting 
and this observation is consistent with the difference in a magnitude of the lift forces on the different sides of the inertial focusing position. The asymmetry of the laminar velocity profile results in a lower pressure near the walls (high-velocity), as compared to the center of the microchannel (low-velocity), leading the particles to migrate away from center of the microchannel until they are repulsed by the walls, because the shear-induced inertial lift force is more dominant than the wall-induced lift force. Therefore, the lift force becomes larger nearer to the channel walls and the particles are pushed to the area between center streamlines and channel walls corresponding to the particles equilibrium position. In addition, the larger particles moved and migrated more easily than smaller particles due to their higher surface area and rotated and were transported more easily in the flowing fluid.

In the present study, the particles in the microchannel tend to migrate toward the wall vicinity area rather than the center of the microchannel as the particles move from the upstream cross-section to the downstream cross-section, where the lowest particle volume fraction occurs at electrode pairs 2-11 and 5-8 at the upstream cross-section and at electrode pairs 3-14 and 4-9 at the downstream cross-section. The results of the particles migration to move to the wall vicinity area is similar with the Serge-Silberberg phenomenon [4], where the particles migrate across the streamlines at the lateral equilibrium position, which is approximately halfway between the center of the microchannel and the walls.

\section{Conclusions}

In the present paper, we investigated the measurement of the particle volume fraction based on cross-sectional capacitance measurement for different electrode pairs at upstream and downstream cross-sections. The particles are distributed non-uniformly due to the inertial lift forces depending on the gradient velocity profile distribution in the microchannel. The results of the present study are as follows:

1. The particle volume fraction at the downstream cross-section is increased to $9 \%$ for electrode pairs near the channel walls and is decreased to less than $1 \%$ for electrode pairs near the center of the microchannel, as compared to the upstream cross-section, because the particles have migrated in the fluid flow due to inertial lift forces and particle velocity.

2. The larger particles were demonstrated to move and migrate more easily than smaller particles due to the high surface interaction area and are easily rotated and transported in the fluid flow.

3. The particle volume fraction revealed that particles migrated toward the channel walls as particles moved from the upstream cross-section to the downstream cross-section.

\section{Acknowledgments}

The present study was supported by the Adaptable and Seamless Technology Transfer Program through Target-driven R\&D (A-Step) of the Japan Science and Technology Agency (\#AS2311054B).

\section{References}

[1] GK Ananthasuresh and S Chakraborty. Micromechanics of engineered and biological systems, Current Trends in Science: Platinum Jubilee Special. 2010.

[2] J Feng and DD Joseph. The motion of particles suspended in viscoelastic under torsional shear. J. Fluid Mech., Vol. 324, 199-222. 1996.

[3] YW Kim and JY Yo. The lateral migration of neutrally-buoyant spheres transported through square microchannels. Journal of Micromechanics and Microengineering, Vol. 18, 065015. 2008.

[4] G Segre and A Silberberg. Behavior of macroscopic rigid spheres in Poiseuille flow: Part 1. Determination of local concentration by statistical analysis of particle passage through crossed light beams. J. Fluid Mech., Vol. 14, 115-135. 1962.

[5] RG Cox and SG Mason. Suspended particles in fluid flow through tubes. Ann. Rev. Fluid Mech., Vol. 3, 291-316. 1971. 
[6] RV Repetti and EF Leonard. Segre-Silberberg annulus formation: A possible explanation Nature, Vol. 203, 1346. 1964.

[7] JS Halow and GB Wills. Radial migration of spherical particles in Couette systems. IChE J., Vol. 16, 281-286. 1970.

[8] DR Oliver. Influence of particle rotation on radial migration in the Poiseuille flow of suspensions. Nature Journal, Vol. 194, 1269-71. 1962.

[9] HL Goldsmith and SG Mason. The flow of suspensions through tubes: I. Single spheres, rods, and discs. J. Colloid Sci., Vol. 17, 448-76. 1962.

[10] RC Jeffrey and JRA Pearson. Particle motion in laminar vertical tube flow. J. Fluid Mech., Vol. 22, 721-35. 1965.

[11] B Chun and AJC Ladd. Inertial migration of neutrally buoyant particles in a square duct: an investigation of multiple equilibrium positions. Phys. Fluids, Vol. 18, 03170. 2006.

[12] BP Ho and LG Leal. Inertial migration of rigid spheres in two-dimensional unidirectional flows. J. Fluid Mech., Vol. 65, 365-400. 1974.

[13] J Feng, HH Hu, and DD Joseph. Direct simulation of initial value problems for the motion of solid bodies in a Newtonian fluid: Part 2: Couette and Poiseuille flows. J. Fluid Mech., Vol. 277, 271-30. 1994.

[14] A Asgar, S Bhagal, S Sathyakumar, Kuntaegowdanahaili, and I Papautsky. Enhanced particle filtration in straight microchannel using shear-modulated inertial migration. Physics of Fluids, 101702. 2008.

[15] J Oakey, RW Applegate, Jr., E Arellano, DD Carlo, SW Graves, and M Tone. Particle focusing in staged inertial microfluidic devices for flow cytometry. Analytical Chemistry, Vol. 82, 3862-3867. 2010.

[16] DS Holder. Electrical impedance tomography: methods, history, and applications. Institute of Physics Publishers, ISBN 0750309520, 9780750309523, 295-307. 2005.

[17] H Griffiths, MG Tucker, I Sage, and WG Hrrenden-Harker. An electrical impedance tomography microscope, Physiology Measurement, Vol. 17, 15-24. 1996.

[18] TA York, TN Phua, L Reichelt, A Pawlowski, and RKneer. A miniature electrical capacitance tomography, Measurement, Vol. 17, 2119-2129. 2006.

[19] J Choi and M Takei. Sub-Micro particle distribution of measurement and simulation in cross-section of microchannel by process computed tomography. IEEE Journal, 41-46. 2010.

[20] A Jaworek and A Krupa. Phase-shift detection for capacitance sensor measuring void fraction in two-phase flow. Journal of Sensors and Actuators, Vol. 160, 78-86. 2010.

[21] J Choi, M Takei, and DH Doh. Fabrication of microchannel with 60 electrodes and capacitance measurement. Flow Measurement and Instrumentation Journal, Vol. 21, 178-183. 2010.

[22] SM Huang, CG Xie, R Thorn, D Snowden, and MS Beck. Design of sensor electronics for electrical capacitance tomography. IEEE Journal, Vol. 139, 83-88. 1992.

[23] WQ Yang and TA York. New AC-based capacitance tomography system. Science, Measurement and Technology Journal, Vol. 146, 47-53. 1999.

[24] M Louge and M Opie. Measurements of the effective dielectric permittivity of suspensions. Journal of Powder Technology, Vol. 62, 85-94. 1990. 


\section{Figure}

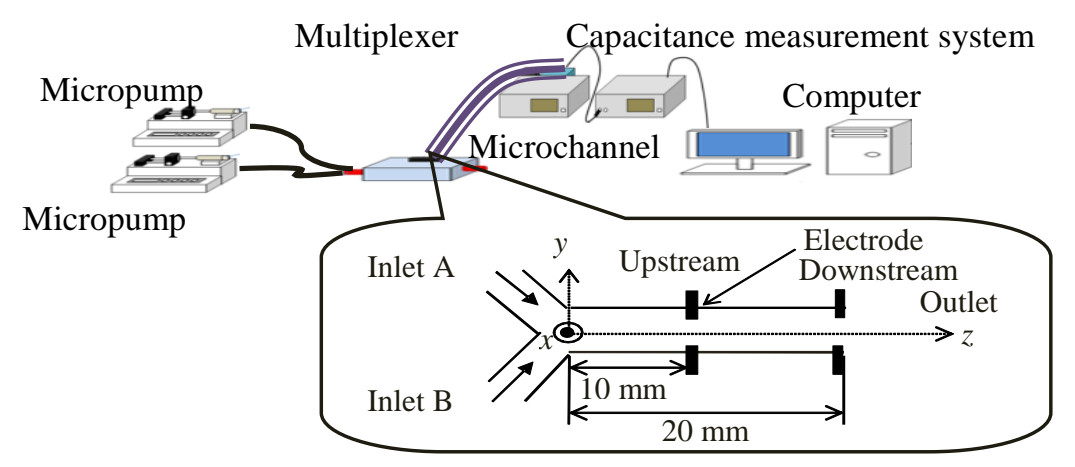

Fig. 1 Experimental set-up for cross-sectional capacitance measurement and locations of the upstream and downstream cross-sections.

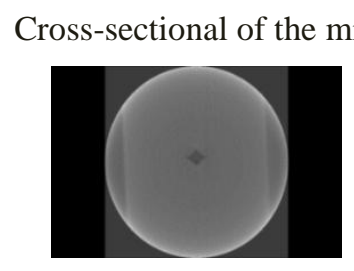

(a)

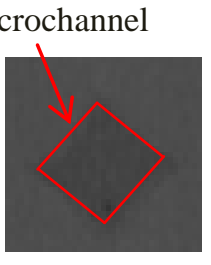

(b)

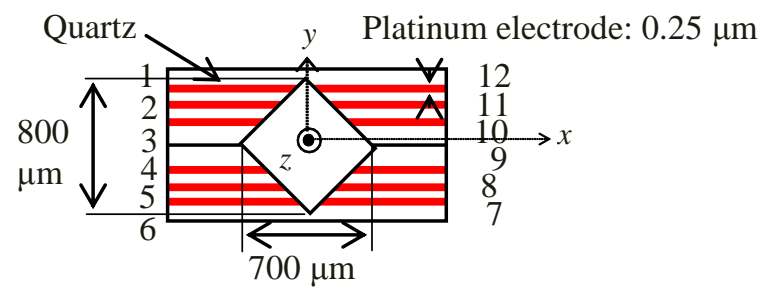

(c)

Fig. 2 (a) Image and (b) close-up image of the diamond-shaped groove of the cross-sectional microchannel captured by high-speed micro-computed tomography. (c) Schematic diagram of the cross-sectional microchannel.

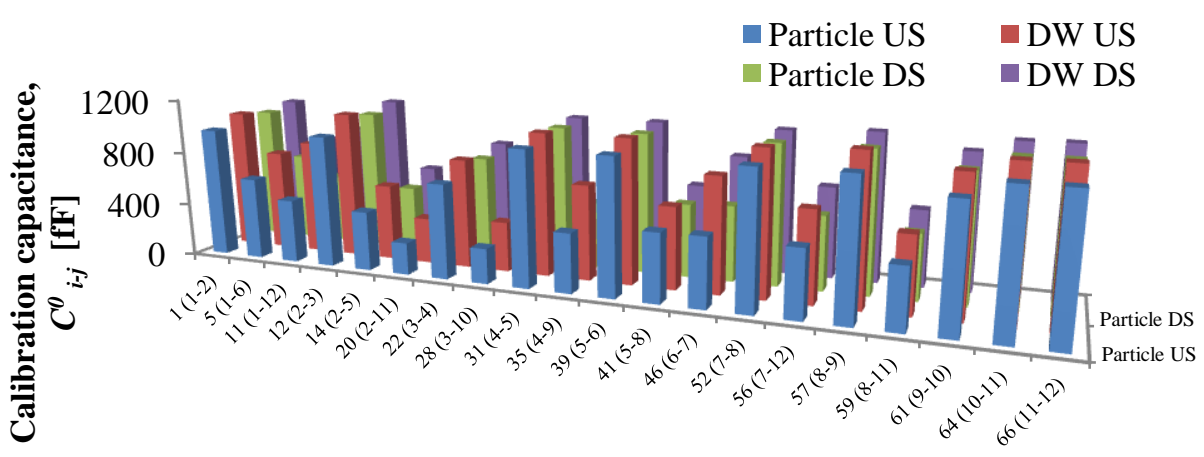

Representative of a number of electrodes (electrode pair)

Fig. 3 Calibration cross-sectional capacitance for deionized water (DW) and static $1.3 \mu \mathrm{m}$ particles at the upstream (US) and downstream (DS) cross-sections. 


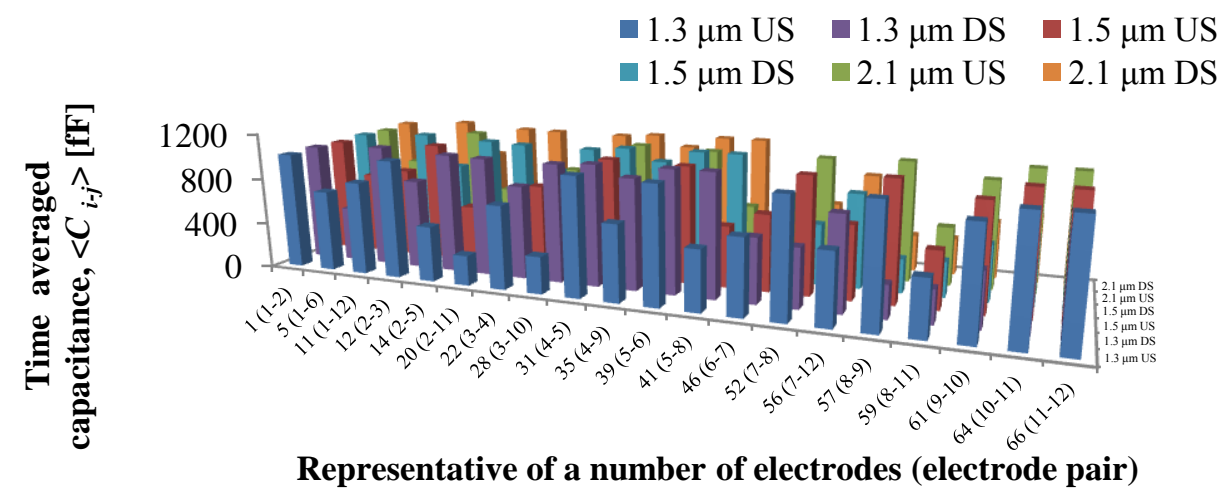

Fig. 4 Time-averaged capacitance for flow particles at the upstream (US) and downstream (DS) cross-sections.

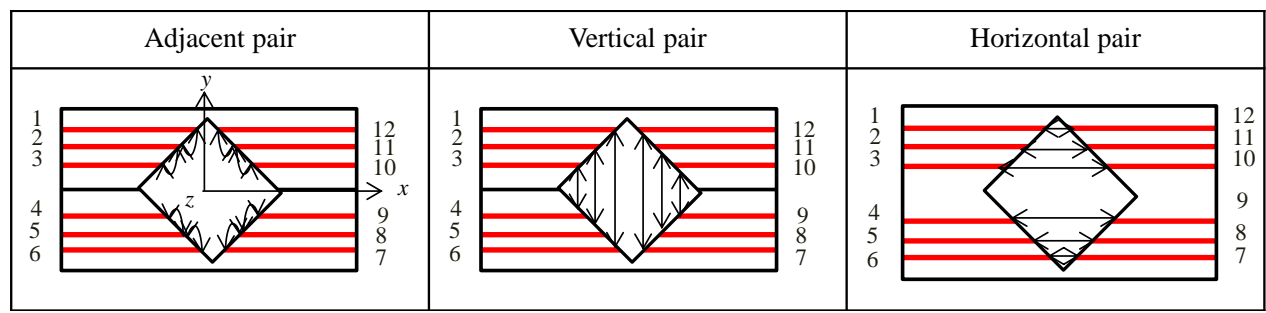

Fig. 5 Area measurement of electrode pairs in the particle volume fraction calculation

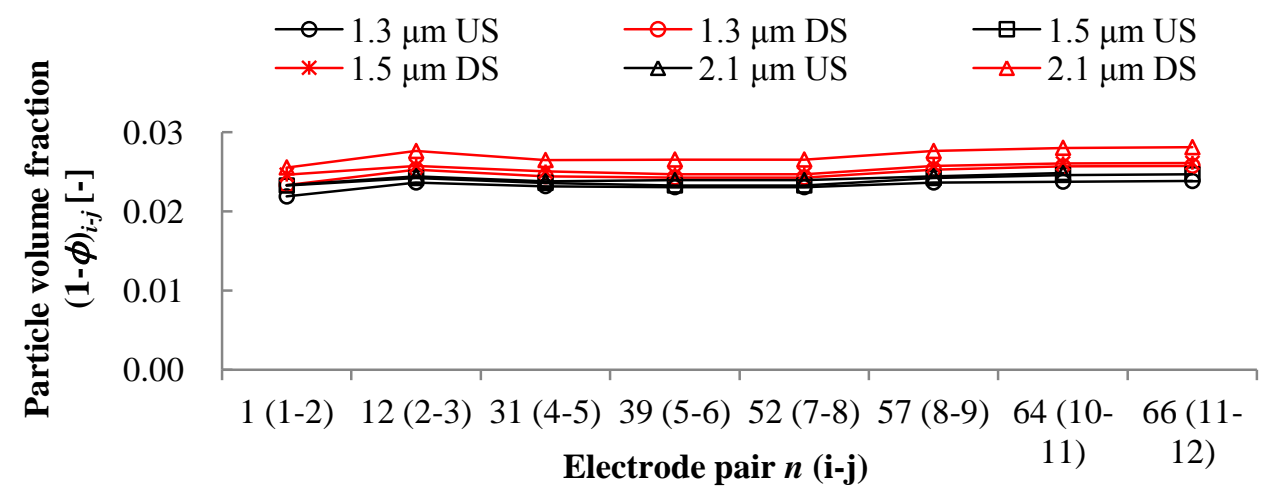

Fig. 6 Particle volume fractions for three particles sizes for an adjacent electrode pair at the upstream (US) and downstream (DS) cross-sections.

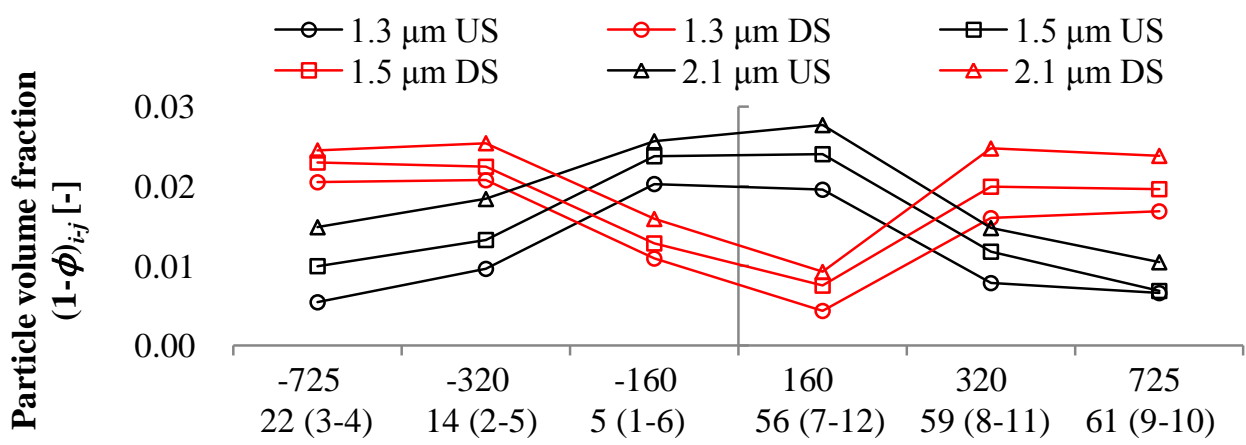

Electrode distance along $\boldsymbol{x}$-axis $[\boldsymbol{\mu m}]$, Electrode pair $\boldsymbol{n}(\mathbf{i}-\mathbf{j})[-]$

Fig. 7 Particle volume fractions for three particles sizes for a vertical electrode pair at the upstream (US) and downstream (DS) cross-sections. 


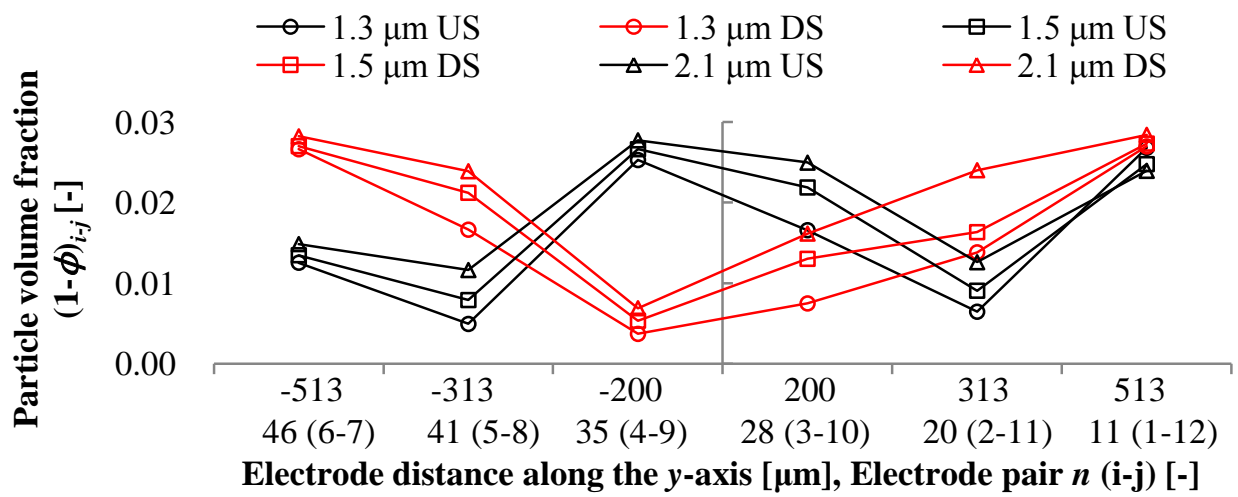

Fig. 8 Particle volume fractions for three particles sizes for a horizontal electrode pair at the upstream (US) and downstream (DS) cross-sections.

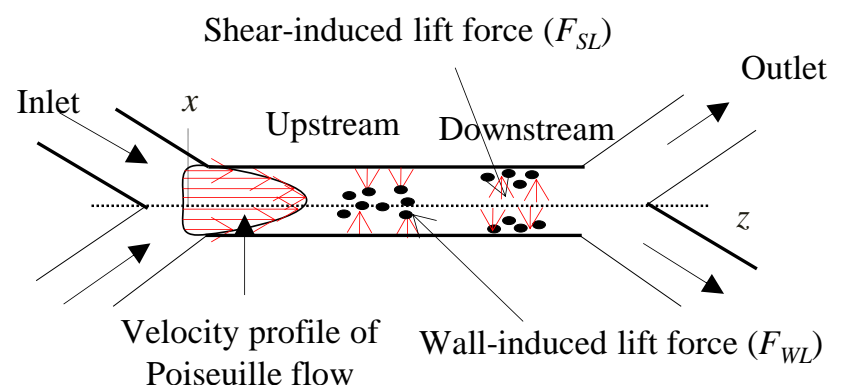

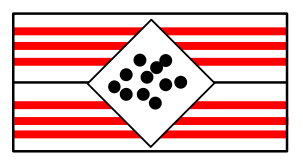

Upstream

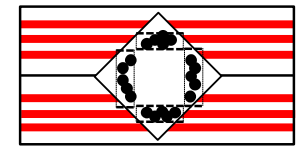

Downstream

Fig. 9 Summary of the particle volume fraction at the upstream and downstream cross-sections. 


\section{Table}

Table 1 Experimental conditions of inlet injection

\begin{tabular}{cccc}
\hline Case & Inlet A & Inlet B & Cross-section \\
\hline 1 & Deionized water & Deionized water & Upstream, Downstream \\
2 & $3 \%$ particles + deionized & $3 \%$ particles + deionized & Upstream, Downstream \\
\hline
\end{tabular}

Table 2 The 66 electrode pairs used in the cross-sectional capacitance measurement

\begin{tabular}{|c|c|c|c|c|c|c|c|c|c|c|c|c|c|}
\hline & \multicolumn{13}{|c|}{ Electrode number (detector) $[j]$} \\
\hline \multirow{13}{*}{$\begin{array}{c}\text { Electrode } \\
\text { number } \\
\text { (source) } \\
{[i]}\end{array}$} & & 1 & 2 & 3 & 4 & 5 & 6 & 7 & 8 & 9 & 10 & 11 & 12 \\
\hline & 1 & $X$ & 1 & 2 & 3 & 4 & 5 & 6 & 7 & 8 & 9 & 10 & $\underline{11}$ \\
\hline & 2 & & $X$ & 12 & 13 & 14 & 15 & 16 & 17 & 18 & 19 & $\underline{20}$ & 21 \\
\hline & 3 & & & $X$ & 22 & 23 & 24 & 25 & 26 & 27 & $\underline{28}$ & 29 & 30 \\
\hline & 4 & & & & $\mathrm{X}$ & 31 & 32 & 33 & 34 & $\underline{35}$ & 36 & 37 & 38 \\
\hline & 5 & & & & & $X$ & 39 & 40 & $\underline{41}$ & 42 & 43 & 44 & 45 \\
\hline & 6 & & & & & & $\mathrm{X}$ & $\underline{46}$ & $\overline{47}$ & 48 & 49 & 50 & 51 \\
\hline & 7 & & & & & & & $\bar{X}$ & 52 & 53 & 54 & 55 & 56 \\
\hline & 8 & & & & & & & & $\mathrm{X}$ & 57 & 58 & 59 & 60 \\
\hline & 9 & & & & & & & & & $\mathrm{X}$ & 61 & 62 & 63 \\
\hline & 10 & & & & & & & & & & $X$ & 64 & 65 \\
\hline & 11 & & & & & & & & & & & $X$ & 66 \\
\hline & 12 & & & & & & & & & & & & $X$ \\
\hline
\end{tabular}

